\title{
Wie entscheidungs- und reformfähig sind demokratische politische Systeme?
}

von Ludger Helms

Die Persistenz und Wohlfahrt demokratischer politischer Systeme hängt in hohem Maße von ihrer Entscheidungs- und Reformfähigkeit ab. Dem grundsätzlichen Problem, politische Mehrheiten für unpopuläre Reformmaßnahmen zusammenzubringen, sehen sich alle demokratischen Systeme gegenüber. In institutioneller Hinsicht sind die Voraussetzungen hierfür in komplexen gewaltenverflochtenen Systemen besonders anspruchsvoll. Rein institutionelle Erklärungen strukturell verminderter Reformfähigkeit von Systemen greifen jedoch ebenso zu kurz wie Ansätze, die ganz auf die Reformscheu oder -müdigkeit der Bürger konzentriert sind. In der entwickelten repräsentativen Demokratie liegt die Hauptverantwortung für die Konzeption und Durchführung notwendiger Reformen bei den regierenden Parteien und Inhabern politischer Führungsämter. Die Substanz ihrer Reformagenden und ihre Fähigkeit zu angemessener Reformkommunikation bestimmen die Reformfähigkeit eines Systems maßgeblich mit. Das gilt nicht nur für unterschiedliche wohlfahrtsstaatliche Reformen, sondern auch für Reformen auf der Ebene der Staatsorganisation und der grundlegenden institutionellen Spielregeln des politischen Wettbewerbs, welche insofern über sich selbst hinausweisen, als sie zugleich die Bedingungen der Reformpolitik in anderen Bereichen nachhaltig mitbestimmen.

The persistence and well-being of democratic political systems depends to a large extent on their ability to allow for decision-making and the successful pursuit of reform policies. The key problem of forging political majorities willing to carry through even unpopular reforms is known to all democratic systems. In complex power-sharing polities this task is particularly difficult to perform. This notwithstanding, purely institutional explanations for a poor reform performance of a given system are just as unconvincing as explanations focusing exclusively on the "reform aversion" of the citizens. In representative democracies, the responsibility for conceptualising and implementing political reforms lies primarily with the governing parties and those holding government offices. The substance of their reform agendas and, in particular, their communication skills have a major impact on the overall reform capacity of democratic systems. This is true not only for reforms at the level of welfare policies but also, and no less importantly, for reforms of the governing machine and the democratic "rules of the game", which co-determine the conditions for political reforms in other areas. 


\section{Einleitung}

Die Überlegenheit liberaler Demokratien gegenüber nicht-demokratischen Regimen in zentralen Fragen der Herrschaftsorganisation und des politischen Prozesses, wie der politischen Selbstbestimmung, der Freiheit und Rechtssicherheit oder des Minderheitenschutzes, steht heute außer Frage. In der jüngeren Literatur erscheinen diese Aspekte als unterschiedliche Komponenten der „politischen Produktivität“ demokratischer Systeme. ${ }^{1}$ Speziellere Studien suggerieren, dass die Möglichkeit zu umfassender demokratischer Beteiligung nicht nur der Qualität von Entscheidungen und der Legitimität politischer Ordnungen zugute kommt, sondern zugleich die allgemeine Lebenszufriedenheit der Mitglieder eines Gemeinwesens zu steigern vermag. ${ }^{2}$ Die globale Ausstrahlungswirkung der Demokratie beruht in hohem Maße auf diesen Leistungen demokratischer politischer Systeme; sie haben eine Inanspruchnahme des Demokratiebegriffs zur Selbstbeschreibung auch zahlreicher nicht-demokratischer Regime bereits lange vor dem vermeintlichen „Ende der Geschichte“ befördert. ${ }^{3}$

Weniger eindeutig ist die Leistungsbilanz von Demokratien auf der policy-Ebene. Bei Vergleichen wohlfahrtsstaatlicher Leistungsprofile von Regimen schneiden demokratische Systeme keineswegs durchgehend signifikant besser ab als nicht-demokratische Regime. Das gilt insbesondere für Leistungsvergleiche zwischen ausgewählten nicht-demokratischen Systemen und wenig konsolidierten Demokratien jenseits der westlichen Hemisphäre. ${ }^{4}$ Noch deutlich umstrittener ist die Entscheidungs- und Reformfähigkeit demokratischer politischer Systeme. Sie kann geradezu als die „Achillesferse“ komplexer liberal-demokratischer Gemeinwesen erscheinen. Es ist diese Frage nach der Entscheidungs- und Reformfähigkeit demokratischer politischer Systeme, die im Mittelpunkt des vorliegenden Beitrags steht.

1 Roller, E.: The Performance of Democracies: Political Institutions and Public Policies, Oxford, 2005.

2 Owen, A./Videras, J./Willemsen, C.: Democracy, Participation, and Life Satisfaction, in: Social Science Journal, 87/4 (2008), 987-1005.

3 Vgl. Conze, W.: „Demokratie“ in der modernen Bewegung, in: Brunner, O./Conze, W./Koselleck, R. (Hg.): Geschichtliche Grundbegriffe. Historisches Lexikon zur politisch-sozialen Sprache in Deutschland, Bd. 1, Stuttgart 1972, 873-899, hier 898. ,Facade democracies “ sind freilich auch nach dem weltgeschichtlichen Umbruch von 1989 außerordentlich weit verbreitet, nach dem Urteil einiger Beobachter sogar auf dem Vormarsch. Vgl. Diamond, L.: The Democratic Rollback, in: Foreign Affairs, 87/2 (2008), 36-48.

4 Schmidt, M. G.: Zur Leistungsfähigkeit von Demokratien - Befunde neuerer vergleichender Analysen, in: Brodocz, A./Llanque, M./Schaal, G. S. (Hg.): Bedrohungen der Demokratie, Wiesbaden, 2008, 2941. 
Angesichts der universellen Relevanz des Themas kann dabei nur eine vergleichende Perspektive zu differenzierenden Einsichten gelangen. Vor allem das Kernproblem, politische Mehrheiten für gesellschaftlich unpopuläre Reformmaßnahmen zusammenzubringen, kennen alle demokratischen Systeme. Das belegt auch die politische Rhetorik in institutionell und politisch hochgradig unterschiedlich beschaffenen Demokratien. Die Einsicht, „Turkeys do not vote for Christmas!" entstammt nicht etwa dem reformpolitischen Erfahrungsschatz leidgeprüfter Modernisierungsbefürworter in der Bundesrepublik oder anderen kontinentaleuropäischen Verhandlungsdemokratien, sondern der britischen Westminster-Demokratie, deren institutionelle Struktur einst treffend als ,a set of arrangements facilitating governance ${ }^{i 5}$ beschrieben wurde.

Diese Abhandlung darf nicht beanspruchen, mehr als einige Hinweise auf notwendige terminologische Differenzierungen, den Stellenwert unterschiedlicher Teilaspekte des Gegenstands und den Zusammenhang zwischen jenen Faktoren zu geben, die in künftigen Diskussionen einer stärkeren Berücksichtigung würdig erscheinen.

\section{Entscheidungsfähigkeit, Reformfähigkeit und die Struktur politischer Reformen}

Notwendig ist zunächst eine Differenzierung zwischen der Entscheidungs- und Reformfähigkeit politischer Systeme. Entscheidungsfähigkeit meint mit Blick auf die Systemdimension die strukturelle Fähigkeit von Systemen, innerhalb der gegebenen konstitutionellen bzw. institutionellen Rahmenbedingungen gesamtgesellschaftlich verbindliche Entscheidungen zu erzeugen. In allen demokratischen Systemen kommt dabei - mit freilich sehr unterschiedlicher Konditionierung - das Mehrheitsprinzip zum Tragen. Die „technische“ Dimension der Entscheidungsfähigkeit demokratischer Systeme ist überall dort gewährleistet, wo es eine absolute Mehrheit bzw., sofern gefordert, eine hinreichend große qualifizierte Mehrheit gibt, auf die sich Entscheidungsakteure in den staatlichen Lenkungsorganen stützen können. Im legislativen Verfahren repräsentativdemokratischer Systeme geht es dabei um eine Mehrheit von Parlamentsmandaten bzw. um eine Mehrheit der Stimmen von Inhabern eines parlamentarischen Mandats. Aufgrund der üblicherweise weitreichenden, institutionell beförderten

5 So Jordan, G.: The British Administrative System: Principles vs. Practice, London/New York, 1994, 169. 
Partei- bzw. Fraktionsdisziplin unter den Abgeordneten parlamentarischer Demokratien ${ }^{6}$ ist das Spannungsverhältnis zwischen numerischen parlamentarischen Mehrheiten und operativen parlamentarischen Entscheidungsmehrheiten in Systemen dieses Typs im Allgemeinen gering ausgeprägt. ${ }^{7}$ Gleichwohl erweist sich die politische Gewährleistung der technischen Entscheidungsfähigkeit parlamentarischer Demokratien zuweilen als ausgesprochen aufwendig. Das gilt schon für Einparteienregierungen und mehr noch für Koalitionsregierungen, ganz besonders jedoch in all jenen Fällen, in denen Regierungen über keinen parlamentarischen Mehrheitsstatus verfügen und parlamentarische Mehrheiten auf ad-hoc-Basis bilden müssen. ${ }^{8}$

Spezifische Konditionierungen des Anwendungs- bzw. Geltungsbereichs des Mehrheitsprinzips betreffen unterschiedliche Ebenen des demokratischen Entscheidungsprozesses. Einige Länder, wie Österreich, kennen eine Einstimmigkeitsregel für Kabinettsbeschlüsse, die nicht allein auf politischen Konventionen beruht, sondern auch von Verfassungsrechtlern ausdrücklich postuliert wird. ${ }^{9}$ Sehr viel verbreiteter sind qualifizierte Mehrheitserfordernisse für Verfassungsänderungen. Die erschwerte Abänderbarkeit von Verfassungsregeln gilt geradezu als ein konstitutives Bestimmungsmerkmal des Verfassungsrechts. Dabei können spezifische parlamentarische Mehrheitserfordernisse (von einer Drei-Fünftel- bis zu einer Zwei-Drittel-Mehrheit) um weitere (etwa direktdemokratische) Zustimmungserfordernisse ergänzt werden. ${ }^{10}$ Daneben gibt es vielerorts zusätzliche institutionelle Barrieren gegen Mehrheitsherrschaft. Dazu zählen vetomächtige Akteure wie Staatsoberhäupter oder Verfassungsgerichte auf der horizontalen Ebene ebenso wie föderative Strukturen als Ausprägungen vertikaler Gewaltenteilung, die die Handlungs- und Gestaltungsmacht zentralstaatlicher Regierungen auf spezifische Weise beschränken. In der vergleichenden Literatur wurde mit

6 Vgl. Bowler, S./Farrell, D.M./Katz, R.S. (Hg.): Party Discipline and Parliamentary Government, Ohio, 1999; Hazan, R. Y. (Hg.): Cohesion and Discipline in Legislatures, London/New York, 2006.

7 Größer ist in vielen Systemen die Differenz zwischen den Stimmen- und Mandatsmehrheiten regierender Parteien, wobei vor allem Systeme mit Mehrheitswahl auf der Grundlage von , manufactured majorities “ operieren. Vgl. Nohlen, D.: Wahlrecht und Parteiensystem, 6. Aufl., Opladen, 2009.

8 Vgl. Kropp, S./Schüttemeyer, S. S./Sturm, R. (Hg.): Koalitionen in West- und Osteuropa, Opladen, 2002; Strøm, K./Müller, W.C./Bergman, T. (Hg.): Cabinets and Coalition Bargaining: The Democratic Life Cycle in Western Europe, Oxford, 2008; Gianetti, D./Bennoit, K. (Hg.): Intra-Party Politics and Coalition Governments, London, 2009.

9 Müller, W.C.: Regierung und Kabinettssystem, in: Dachs, H. u.a. (Hg.): Politik in Österreich, Wien, 2006, 168-187, hier $175 \mathrm{f}$.

10 Lutz, D. S.: Toward a Theory of Constitutional Amendment, in: American Political Science Review, 88/2 (1994), 355-370. 
Blick auf diese Dimension demokratischer Systeme nach Regimen mit unterschiedlichen Graden des institutionellen Pluralismus differenziert; ${ }^{11}$ ihr korrespondiert die griffigere Unterscheidung zwischen „simple polities“ und „compound polities ". ${ }^{12}$ Die Einsicht in die entscheidungspolitische Relevanz dieser Institutionen und Akteure ist bedeutend älter als das Vetospieler-Theorem ${ }^{13}$, welches die klassische Regierungsformen- und Systemlehre gezielt herausfordert (vgl. Abschnitt III).

Betrachtungen zur Entscheidungsfähigkeit politischer Systeme setzen in der Regel voraus, dass eine adäquate Umsetzung von rechtmäßig zustande gekommenen Entscheidungen auf jeden Fall gewährleistet ist. Dies ist jedoch keineswegs immer, zumindest nicht stets in gleichem Maße der Fall. Die „Verschleppung“ oder „Verwässerung“ von politischen Entscheidungen im Implementationsstadium besitzt alles andere als Seltenheitswert wie nicht erst die Europaforschung mit ihren Beiträgen zu den Problemen der nationalstaatlichen Umsetzung von EU-Entscheidungen zutage gefördert hat. Die Umsetzung politischer Entscheidungen, besonders von politisch brisanten Reformmaßnahmen, bedarf deshalb der gesonderten Aufmerksamkeit. Längst ist die Forschung mit guten Gründen dazu übergegangen, den Fokus dabei nicht ausschließlich auf das Tun und Lassen der staatlichen Verwaltung zu beschränken, sondern auch die Rolle gesellschaftlicher Akteure angemessen zu würdigen. ${ }^{14}$

Reformfähigkeit reicht vom Anspruch her über die bloße Entscheidungsfähigkeit politischer Systeme hinaus. Gefordert ist, dass die strukturelle Entscheidungsfähigkeit (soweit gegeben) in den Dienst einer spezielleren reformpolitischen Agenda gestellt wird. In diesem Sinne setzt Reformfähigkeit des politischen Systems die Fähigkeit und den Willen der maßgeblichen Entscheidungsakteure des Systems zur Planung und Umsetzung politischer Reformen voraus. Das erscheint in theoretisch-konzeptueller Hinsicht geradezu banal, mit Blick auf die politische

11 Colomer, J.P.: Introduction, in: ders. (Hg.): Political Institutions in Europe, London/New York, 1996, 1-17, hier 9 .

12 Schmidt, V.A.: Democracy in Europe: The Impact of European Integration, in: Perspectives on Politics, 3/4 (2005), 761-779, hier $762 \mathrm{f}$.

13 Tsebelis, G.: Veto Players: How Political Institutions Work, Princeton, 2002.

14 Mayntz, R.: Implementation politischer Programme: Empirische Forschungsberichte, Königstein/Ts., 1980; dies.: Implementation politischer Programme: Ansätze zur Theoriebildung, Königstein/Ts., 1983; Sabatier, P.A./Mazmanian, D.A.: Implementation and Public Policy, Lanham, MD, 1989; Hill, M./Hupe, P.: Implementing Public Policy: Governance in Theory and Practice, London, 2002; dies.: Implementing Public Policy: An Introduction to the Study of Operational Governance, 2. Aufl., London, 2009. 
Dimension dieser Bedingung hingegen keineswegs. Dazu weiter unten mehr. Schon an dieser Stelle sei angemerkt, dass es freilich sehr unterschiedlich weitreichende Vorstellungen darüber gibt, worauf sich Reformfähigkeit nun eigentlich bezieht. Expansive Konzepte möchten darunter nicht lediglich „die Frage nach adäquaten Steuerungsinstrumenten und der Aufarbeitung von Rationalitätsdefiziten in Staat und Verwaltung“ verstehen, ,sondern auch [... nach ...] Weiterentwicklung der sozialen Strukturen in Richtung einer gesellschaftsunmittelbaren Problemlösungsfähigkeit" ${ }^{\star 15}$. Dabei geht es offenkundig um die Ausdehnung des Reformbegriffs von der Staats- und Politikreform auf die Demokratie- und Gesellschaftsreform.

Gleichsam gedanklich vorzuschalten ist der Reflexion über die Reformfähigkeit demokratischer Systeme eine Verständigung über den Begriff der politischen Reform selbst. Klaus Dieter Wolf versteht darunter ,durch politische Entscheidungen bewusst herbeigeführte Veränderungen von Strukturen, Verfahren und Programmen “16. Manfred G. Schmidt fügt diesem Verständnis das Kriterium des Einsatzes von ausschließlich „legalen Mitteln“"17 hinzu, wodurch der Begriff „Reform“ von jenem der „Revolution“ abgesetzt ist. Bei einigen Autoren erscheint der kategorische Ausschluss revolutionärer Aktivitäten gar gleichbedeutend mit einem ausgeprägt inkrementalen Charakter politischer Reformen ${ }^{18}$, der zweifelsohne möglich und verbreitet, gegebenenfalls auch sinnvoll, aber dennoch weder terminologisch noch konzeptuell zwingend ist.

Beachtung verdient auch die Unterscheidung zwischen unterschiedlichen Kernbereichen staatlicher Reformpolitik: Reformen an den staatlichen Institutionen einerseits und jenen auf der Ebene materieller Politiken andererseits. Sie sind weniger stark voneinander geschieden als in der älteren Literatur vielfach suggeriert wurde. Zunächst ist daran zu erinnern, dass die Reform staatlicher Institutionen keineswegs vollständig außerhalb des Rahmenkonzepts der Staatstätigkeit steht. Das verdeutlicht der wenig gebrauchte, aber nichtsdestoweniger treffsiche-

15 So Blöcker, A./Heyder, U./Mangels-Voegt, B.: Einleitung: Bedingungen für Reformen in den 90er Jahren, in: dies. (Hg.): Die Reformfähigkeit von Staat und Gesellschaft, Frankfurt/M. u. a., 1997, 13-26, hier 14.

16 Wolf, K. D.: Einführungsvortrag: Staat und Gesellschaft - fähig zur Reform?, in: ders. (Hg.): Staat und Gesellschaft - fähig zur Reform?, Baden-Baden, 2007, 19-33, hier 5.

17 Schmidt, M. G.: Wörterbuch zur Politik, 2. Aufl., Stuttgart, 2004, 597.

18 „Reformpolitik vollzieht sich immer in kleinen kontrollierbaren und korrigierbaren Schritten“; so Blöcker, A./Heyder, U./Mangels-Voegt, B., a.a.O., 13. 
re Begriff der „Institutionspolitik“ ${ }^{19}$ Ihm korrespondiert der stärker zur Gesellschaft hin geöffnete Begriff der „Demokratiepolitik“. ${ }^{20}$

In der jüngeren Literatur stößt man gelegentlich auf konzeptuelle Spitzfindigkeiten, die einem analytischen Zugriff auf unterschiedliche Ebenen reformpolitischer Aktivitäten demokratischer Systeme wenig zuträglich sind. Vereinzelt erscheint die klassische Unterscheidung zwischen polity und policy in Teilen der neo-institutionalistischen Literatur gar vollständig aufgehoben, indem policies ausdrücklich als institutions begriffen werden. So konstatiert etwa Paul Pierson: „It makes good sense to think of the major public policies as important institutions. For the individuals and social organizations that make up civil society public policies are clearly very central rules governing their interactions. ${ }^{، 21}$ Ein kaum geringeres Diffusionspotential bergen einige Institutionenverständnisse aus der Geschichtswissenschaft, welche unter Institutionen „nicht nur Strukturen, sondern auch Prozesse" fassen. ${ }^{22}$

Vor allem mit Blick auf institutionell komplexe Systeme wie die Bundesrepublik ist der Zusammenhang zwischen Reformen an der Staatsorganisation und politischen Reformen auf der Ebene einzelner Politikfelder nicht ausschließlich von konzeptueller, sondern zugleich von direkter politischer Relevanz: „Da gesellschaftliche und ökonomische Reformen ohne Politik nicht zu erreichen sind, ist die Identifizierung von institutionellen Restriktionen der Reformpolitik [...] Anlaß zur Suche nach institutionellen Reformen, die die Restriktionen zumindest teilweise beseitigen oder umgehen und eine aktivere Politik erlauben.، ${ }^{، 23}$

19 Hesse, J.J./Benz, A.: Die Modernisierung der Staatsorganisation. Institutionspolitik im internationalen Vergleich: USA, Großbritannien, Frankreich, Bundesrepublik Deutschland, Baden-Baden, 1990.

20 Greven, M. T.: Sind Demokratien reformierbar? Bedarf, Bedingungen und normative Orientierungen für eine Demokratiereform, in: Offe, C. (Hg.): Demokratisierung der Demokratie, Frankfurt/New York, 2003, 72-91, hier 76.

21 Pierson, P.: Politics in Time: History, Institutions and Social Analysis, Princeton, 2004, 165; vgl. ders.: Public Policies as Institutions, in: Shapiro, I./Skowronek, S./Galvin, D. (Hg.): Rethinking Political Institutions: The Art of the State, New York, 2006, 114-131.

22 Reinhard, $W$.: Geschichte der Staatsgewalt: eine vergleichende Verfassungsgeschichte Europas von den Anfängen bis zur Gegenwart, Sonderausgabe, München, 2000, $125 \mathrm{f}$.

23 So Bentele, K./Reissert, B./Schettkat, R.: Einleitung: Die Reformfähigkeit von Industriegesellschaften, in: dies. (Hg.): Die Reformfähigkeit von Industriegesellschaften, Frankfurt/New York, 1995, 9-19, hier 17. Entgegen einer verbreiteten (fach-)öffentlichen Wahrnehmung kommt eine aktuelle empirische Analyse jedoch zu dem Ergebnis, dass die Änderungsrate des Grundgesetzes im internationalen Langzeitvergleich keineswegs besonders gering, sondern durchschnittlich hoch ausfällt. Dieser Befund ist auch für die ,policy“-bezogene Reformdiskussion von Relevanz. „Keinesfalls [...] kann das Grundgesetz im Falle ausbleibender oder zu magerer Reformen für die Defizite innerhalb der politischen Arena - wie die Unwilligkeit Mehrheiten zu formieren - verantwortlich gemacht werden“ (Grasl, M./Detzer, S.: Das 
Studien zu den Einstellungen von Bürgern gegenüber politischen Reformen sind ganz überwiegend auf reformpolitische Maßnahmen in der Wohlfahrtspolitik konzentriert. Diese wissenschaftliche Fokussierung entspricht dem sehr unterschiedlich großen gesellschaftlichen Interesse an politischen Reformen in unterschiedlichen Bereichen. Selbst weitreichende Reformen der Staatsorganisation haben die Gemüter der Bevölkerung nie in vergleichbarem Maße erregt wie Reformen wohlfahrtsstaatlicher Programme. Ausnahmen betreffen noch am ehesten „Totalrevisionen“ von Verfassungen. Aber selbst tiefe Einschnitte in der Verfassungsentwicklung eines Gemeinwesens gehen nicht selten an weiten Teilen der Bevölkerung vorbei. Das gilt besonders für Verfassungsschöpfungen und -reformen im Gefolge schwerer politischer und ökonomischer Krisen. Nach einer Untersuchung des Instituts für Demoskopie vom März 1949 in Westdeutschland beispielsweise zeigten lediglich $21 \%$ der Befragten ein großes Interesse, $33 \%$ hingegen waren nur wenig und $40 \%$ überhaupt nicht an der Arbeit des Parlamentarischen Rates interessiert. ${ }^{24}$ Andere Studien belegen, dass die Existenz des am 24. Mai 1949 in Kraft getretenen Grundgesetzes noch im Dezember 1949 weniger als $40 \%$ der Einwohner der Amerikanischen Zone bekannt war. ${ }^{25}$

In der öffentlichen Reformdiskussion - und nicht nur dort ${ }^{26}$ - werden Reformen auf der Ebene materieller Politiken weithin mit restriktiven oder redistributiven Strukturveränderungen gleichgesetzt. Das Spektrum denkbarer reformpolitischer Maßnahmen ist jedoch bedeutend weiter dimensioniert. Schon die frühe Unterscheidung Theodore Lowis aus den sechziger Jahren zwischen distributiven, redistributiven und regulatorischen policy-Typen ging über eine simple Zweiteilung von Maßnahmen hinaus. ${ }^{27}$ Als die wichtigste Referenztypologie der jüngeren Literatur kann heute die Unterscheidung Klaus von Beymes gelten: Auf der regulativen Ebene wird dabei zwischen restriktiven, regulativen und expansiven

Grundgesetz im Wandel - Die institutionelle Reformfähigkeit Deutschlands im internationalen Vergleich, in: Wagschal, U. (Hg.): Deutschland zwischen Reformstau und Veränderung, Baden-Baden, 2009, 227-248, hier 247.

24 Vgl Recker, M.-L.: Die Verabschiedung des Grundgesetzes, in: Der Bürger im Staat, 59/1 (2009), 4-10, hier 10 .

25 Vgl. Rudzio, W.: Das politische System der Bundesrepublik Deutschland, 7. Aufl., Wiesbaden, $2006,50$.

26 Entsprechendes gilt auch für große Teile der statistisch-empirischen Forschung zur Reformfähigkeit politischer Systeme. Viele der international maßgeblichen Reformfähigkeitsindizes basieren auf einer ausgeprägt marktliberalen Weltsicht, die in politischer Hinsicht nicht als Maß aller Dinge angesehen werden kann. Vgl. Jäckle, S.: Deutschland im Vergleich internationaler Reformindizes, in: Wagschal, U. (Hg.), a. a. O., 33-58, hier 58.

27 Vgl. Lowi, T.J.: American Business, Public Policy, Case Studies, and Political Theory, in: World Politics, 16/4 (1964), 677-715. 
Regulierungen, auf der Leistungs- bzw. Verteilungsebene hingegen zwischen protektiven, distributiven und redistributiven Maßnahmen unterschieden. ${ }^{28}$

Je nach konkretem Inhalt einer Entscheidung können sämtliche Entscheidungstypen politisch heftig umstritten sein und mächtige Reformgegner auf den Plan rufen. Das gilt auch für vermeintlich konfliktarme protektive und expansive Maßnahmen, wie die heftigen Auseinandersetzungen über Regelungen zum Schutz ungeborenen Lebens oder Diskussionen über die Einführung des „Ausländerwahlrechts“" in mehreren Ländern belegen. Bei näherer Betrachtung können selbst distributive Maßnahmen, bei denen es gemessen am status quo ante keine wirklichen Verlierer gibt, hochgradig umstritten sein, wie Konflikte über Steuersenkungsreformen zeigen. Als konfliktträchtig erweist sich bei ihnen einerseits die Tatsache, dass es für unterschiedliche Gruppen üblicherweise zu unterschiedlich großen relativen Verbesserungen kommt. Andererseits werden klassische Verteilungsargumente (nicht nur) auf dem Feld der Finanzpolitik seit geraumer Zeit um das Argument der „Generationengerechtigkeit“ ergänzt, mit dem in Gestalt künftiger Generationen gleichsam ein weiterer Akteur hinzugetreten ist, dessen Ansprüchen gebührend Rechnung zu tragen ist. ${ }^{29}$

Das Politisierungspotential einer Entscheidung und ihr öffentlich wahrgenommener Charakter als einschneidende Reformmaßnahme hat viel mit dem Ausmaß der Veränderung des status quo zu tun, das mit ihr erreicht wird. Die politikwissenschaftliche Gesetzgebungsforschung konnte zeigen, dass die formale Kennzeichnung einer Maßnahme als „Änderungsgesetz“ keinen zuverlässigen Anhaltspunkt für das Innovations- bzw. Reformpotential einer Maßnahme bietet. In der Praxis können auch bloße „Novellen“ ein außerordentlich weitreichendes Veränderungspotential besitzen, welches eine Klassifikation als echte Reformmaßnahme rechtfertigt. ${ }^{30}$

\section{Welche Faktoren entscheiden über die Reformfähigkeit demokratischer politischer Systeme?}

Die jüngere Forschung, besonders in Deutschland, neigt dazu, die offensichtlichen Schwierigkeiten der Reform politischer Strukturen, Verfahren und Pro-

28 Beyme, K. v.: Der Gesetzgeber, Opladen, 1997, 37.

29 Vgl. etwa Dethloff, N.: Generationengerechtigkeit als Verteilungsgerechtigkeit, in: Zypries, B. (Hg.): Verfassung der Zukunft, Berlin, 2009, 132-149.

30 Von den 150 wichtigsten Schlüsselentscheidungen des Bundestages der Jahre 1949 bis 1994 waren immerhin „25 formal und 46 de facto Novellen älterer Gesetze“; so Beyme, K. v., a. a. O., 63. 
gramme mit institutionellen Faktoren und der ausgeprägten Reformscheu der Bürger zu erklären. Damit sind in der Tat zwei Variablen bezeichnet, die im Rahmen einer Bewertung der Reformfähigkeit von politischen Systemen unbedingt zu berücksichtigen sind - auch, um die Grundlagen eines fairen Vergleichs der reformpolitischen Leistungsbilanzen von Regierungen unterschiedlicher Systeme zu gewährleisten. Gefordert ist allerdings eine Betrachtung dieser Faktoren mit Augenmaß.

Aus Sicht der „realistischen“ Parlamentarismustheorie darf nicht erwartet werden, dass die Wähler bzw. einzelne Abgeordnete als die eigentlichen Impulsgeber staatlicher Politik auftreten. Politische Führung impliziert in hohem Maße "representation from above", das Werben konkurrierender Parteien um die Zustimmung zu ihren jeweiligen Programmen und regierungspolitischen Agenden. ${ }^{31}$ Die Hauptschuld an einem möglichen Reformversagen träfe die Wähler aus dieser Perspektive nur dann, wenn sie reformorientierten Kräften grundsätzlich die erforderliche politische Unterstützung versagen und stattdessen das Festhalten politischer Parteien am status quo ausdrücklich honorieren würden. Das ist ohne Zweifel von Land zu Land in sehr unterschiedlichem Maße der Fall; die Risiko- und Veränderungsbereitschaft von Gesellschaften variiert erheblich. $\mathrm{Zu}$ Recht wird deshalb die „politisch-soziale Kultur“ zu den zentralen Bestimmungsfaktoren der Reformfähigkeit demokratischer politischer Gemeinwesen gezählt. ${ }^{32}$ Mit Blick auf Deutschland konstatierten Beobachter in diesem Kontext eine ausgeprägte „Sicherheitsfixierung der Wähler, die die Politiker vor kühneren Reformvorhaben zurückschrecken lässt‘ ${ }^{\star 33}$.

Als gleichsam „natürliche“ Eigenschaft von Wählern in der Demokratie wird man mangelnde Veränderungs- und Risikobereitschaft jedoch kaum bezeichnen wollen. Ebenso wenig kann es überzeugen, die in den meisten Demokratien Westeuropas deutlich gewachsene elektorale Volatilität ohne Weiteres als Verfall einer substantiellen demokratischen Wahlkultur zu deuten. Die Zunahme an

31 Schütt-Wetschky, E.: Haben wir eine akzeptable Parlamentarismustheorie?, in: Hartmann, J./Thaysen, U. (Hg.): Pluralismus und Parlamentarismus in Theorie und Praxis, Opladen, 1992, 91-112, hier 95 f.; Andeweg, R. B./Thomassen, J.J.A.: Modes of Political Representation: Toward a New Typology, in: Legislative Studies Quarterly, 30/4 (2005), 507-528, hier 511.

32 Vgl. Oberreuter, H.: Reform in der Demokratie, in: Vereinigung der Bayerischen Wirtschaft e. V. u.a. (Hg.): Einsichten schaffen Aussichten: Die Zukunft der sozialen Marktwirtschaft, Köln, 2008, 330-338, hier 304-305.

33 So Schmidt, M. G.: Das politische System Deutschlands, München, 2007, 477; vgl. in diesem Sinne auch Pulzer, P.: „Modell Deutschland“ - aus angelsächsischer Sicht, in: Hertfelder, T./Rödder, A. (Hg.): Modell Deutschland: Erfolgsgeschichte oder Illusion?, Göttingen, 2007, 190-204, hier 199 f. 
Wechselwahlbereitschaft indiziert, jedenfalls zum Teil, einen Bedeutungsgewinn der den Parteien zugeschriebenen Problemlösungskompetenz als Motiv der Wahlentscheidung, der mindestens so sehr als Zeugnis demokratischer Reife und Mündigkeit gelten kann wie die Alternative eines unerschütterlichen Festhaltens von Wählern an gewachsenen Parteibindungen. ${ }^{34}$ Auch kann aus der erhöhten Wechselwahlbereitschaft von Bürgern nicht auf eine vollständige Austauschbarkeit des parteipolitischen Angebots geschlossen werden. Trotz eines seit Jahrzehnten zu beobachtenden Annäherungsprozesses besitzen die Parteien der europäischen Demokratien unterschiedliche programmatische Profile. ${ }^{35}$

Der Konkurrenz unterschiedlicher Parteien in der elektoralen Arena korrespondiert in der parlamentarischen Demokratie die Annahme, dass die parteipolitische Zusammensetzung der Regierung einen Einfluss auf die inhaltliche Dimension der Staatstätigkeit besitzt. Die normativ-theoretische Erwartung mit Blick auf diesen Zusammenhang lautet: „parties do matter! “. Die Politikwissenschaft hat die Angemessenheit dieser Vermutung in zahlreichen Studien bestätigt, dabei jedoch vor allem auf die institutionelle Konditionierung von party government aufmerksam gemacht. Je größer der Grad des ,institutionellen Pluralismus“ eines Regierungssystems, umso stärker werden die Gestaltungs- und Reformagenden regierender Parteien institutionell und politisch gebrochen. ${ }^{36}$

Auch an diesem Punkt ist jedoch Differenzierung angezeigt: Nicht jede institutionelle Barriere gegen repräsentativdemokratische Mehrheitsherrschaft verdient es gleichermaßen, als Reformhemmnis bezeichnet zu werden. Auf theoretischer Ebene wurde dem in Beiträgen über ,konstruktive Vetospieler“ Rechnung getragen. Diese können unter Umständen erfolgreiche Reformen gar begünstigen, indem sie Reformprotagonisten dazu bringen, die Vorbehalte möglicher Vetospieler zu berücksichtigen, indem sie dabei behilflich sind, mögliche Wirkungen von Reformen abzuschätzen und schließlich, indem sie Reformen irreversibel

34 Vgl. Lane, J.-E./Ersson, S.: Party System Instability in Europe, in: Democratization, 14/1 (2007), 92110.

35 Volkens, A./Klingemann, H.-D.: Parties, Ideologies and Issues. Stability and Change in Fifteen European Party Systems 1945-1998, in: Luther, K.R./Müller-Rommel, F. (Hg.): Political Parties in the New Europe, Oxford, 2005, 143-167.

36 Vgl. Beyme, K. v.: Do Parties Matter? The Impact of Parties on the Key Decisions in the Political System, in: Government \& Opposition, 19/1 (1984), 5-29; Schmidt, M. G.: When Parties Matter: A Review of the Possibilities and Limits of Partisan Influence on Public Policy, in: European Journal of Political Research, 30/2 (1996), 155-184; ders.: The Impact of Political Parties, Constitutional Structures and Veto Players on Public Policy, in: Keman, H. (Hg.): Comparative Democratic Politics, London, 2002, 166-184. 
machen. ${ }^{37}$ Diese Einsicht hat zu Recht Eingang in die konzeptuelle Ausgestaltung von Indizes gefunden, denen es um die statistische Abschätzung der Reformfähigkeit von OECD-Staaten geht. ${ }^{38}$

Empirische Studien bestätigen das Potential unterschiedlicher „Mitregenten“, die Reformfähigkeit politischer Systeme zu erhöhen. Vor allem die möglichen positiven Effekte starker Kontrollressourcen parlamentarischer Versammlungen auf die Reformfähigkeit von Systemen - und ganz besonders auf die Qualität reformpolitischer Maßnahmen - werden mittlerweile von den wenigsten Beobachtern grundsätzlich bestritten. Als entscheidend gelten dabei insbesondere Strukturen, die eine systematische Einbeziehung der Expertise und Ressourcen der Mehrheitsfraktionen begünstigen, deren Mitregierungspotential in der Parlamentarismusliteratur unter dem Stichwort des ,,intra-party mode " diskutiert wird. ${ }^{39}$ Selbst zweite Kammern agieren keineswegs ausschließlich als destruktive oder konstruktive Vetospieler, sondern können ebenso gut als Initiatoren politischer Reformen auftreten. ${ }^{40}$

Anstöße zu politischen Reformen in der Demokratie sind jedoch nicht ausschließlich demokratischen Institutionen bzw. Akteuren im engeren Sinne vorbehalten. Insbesondere Verfassungsgerichte sind in der Verfassungspraxis liberaler Demokratien nicht auf die Rolle einer „Reformbremse“ festgelegt. Vielfach scheint sogar das Gegenteil der Fall zu sein. Im deutschen Kontext wurde die „antizyklische“ Urteilspolitik des Bundesverfassungsgerichts als wohltuender Impuls gegen den „Attentismus der Parteiendemokratie“ gewürdigt. ${ }^{41}$ Die weiterreichende These, nach der die Akteure des politischen Entscheidungssystems

37 Vgl. Benz, A.: Konstruktive Vetospieler in Mehrebenensystemen, in: Mayntz, R./Streeck, W. (Hg.): Die Reformierbarkeit der Demokratie, Frankfurt/New York, 2003, 205-238, hier 230.

38 Vgl. Brusis, M.: Reformfähigkeit messen? Konzeptionelle Überlegungen zu einem Reformfähigkeitsindex für OECD-Staaten, in: Politische Vierteljahresschrift, 49/1 (2008), 92-113. Im Zentrum stehen dabei die erzielten Politikergebnisse und die Regierungsführung, konzeptualisiert als das Zusammenwirken der Regierung mit Akteuren im Regierungsumfeld.

39 Vgl. King, A.: Modes of Executive-Legislative Relations: Great Britain, France, and West Germany, in: Legislative Studies Quarterly, $1 / 1$ (1976), 37-65; Norton, P.: Introduction: The Institution of Parliaments, in: ders. (Hg.): Parliaments and Governments in Western Europe, London, 1998, 1-15.

40 Das gilt auch für den deutschen Bundesrat. Gemäß der großen Studie von Beymes verdankten einige der wichtigsten 150 Schlüsselentscheidungen des Deutschen Bundestages im Zeitraum 1949 bis 1994 ihre Initiative dem Bundesrat; vgl. Beyme, K. v.: Der Gesetzgeber, a. a. O., 378-401.

41 So Guggenberger, B.: Die Rechtsprechung des Bundesverfassungsgerichts und die institutionelle Balance des demokratischen Verfassungsstaates, in: Guggenberger, B./Würtenberger, T. (Hg.): Hüter der Verfassung oder Lenker der Politik? Das Bundesverfassungsgericht im Widerstreit, Baden-Baden, 1998, 202-232, hier 210 . 
die Verfassungsgerichtsbarkeit geradezu dazu missbrauchen, in bestimmten Entscheidungssituationen die Verantwortung für die Initiierung gesellschaftlich unpopulärer Reformpolitiken zu übernehmen, ist plausibel, kann jedoch leicht überstrapaziert werden. ${ }^{42}$

In Situationen, in denen der Reformwille der Bevölkerung größer ist als derjenige der politischen Klasse, können sich direktdemokratische Instrumente als bedeutende zusätzliche Kanäle von Reformpolitik erweisen. Das abrogative Referendum in Italien, mit dem 1993 der Wechsel des Wahlsystems und das Ende des alten Systems der ",partitocrazia“ eingeleitet wurden, gilt als ein westeuropäisches Vorzeigebeispiel der reformpolitischen Wirksamkeit direktdemokratischer Instrumente. ${ }^{43}$ Auch mit Blick auf die reformpolitische Relevanz unterschiedlicher direktdemokratischer Verfahren ist jedoch zu differenzieren: Grundsätzlich besitzen Volksinitiativen eher den Charakter eines „Gaspedals“, während Referenden häufig eher die Funktion eines „Bremspedals“ im politischen Entscheidungsprozess zukommt. ${ }^{44}$

Über der gebührenden Würdigung institutioneller Bestimmungsfaktoren der Reformfähigkeit demokratischer Systeme darf indes nicht vergessen werden, dass zu den notwendigen Voraussetzungen staatlicher Reformpolitik Entscheidungsakteure mit substantiellen Reformagenden gehören. So einsichtig dies in theoretischer Hinsicht ist, so umstritten ist die Fähigkeit und Bereitschaft regierender Parteien und deren Spitzenrepräsentanten zur gezielten Verfolgung politischer Reformen in der Praxis. Besondere Aufmerksamkeit hat in der jüngeren Vergangenheit die Frage nach der Reformfähigkeit sozialdemokratischer Parteien bzw. von diesen geführter Regierungen gefunden. ${ }^{45}$ Die Relevanz der Frage nach der Reformfähigkeit und -willigkeit entscheidungsbefugter Akteure ist freilich nicht auf dieses Spektrum der Parteienlandschaft liberaler Demokratien

42 Vgl. im deutschen Kontext Limbach, J.: Mißbrauch des Bundesverfassungsgerichts durch die Politik?, in: Gegenwartskunde, 48/1 (1999), 11-18.

43 Vgl. Katz, R.: Electoral Reform in Italy: Expectations and Results, in: Acta Politica, 41/3 (2006), 285299.

44 So im schweizerischen Kontext Linder, $W$ : : Schweizerische Demokratie: Institutionen, Prozesse, Perspektiven, Bern, 1999, 259.

45 Vgl. etwa Merkel, W. u.a.: Die Reformfähigkeit der Sozialdemokratie, Wiesbaden, 2006; Egle, C.: Reformpolitik in Deutschland und Frankreich: Wirtschafts- und Sozialpolitik bürgerlicher und sozialdemokratischer Regierungen seit Mitte der neunziger Jahre, Wiesbaden, 2009. 
beschränkt, sondern betrifft grundsätzlich die Vertreter sämtlicher „Parteifamilien“. 46

Es hat immer wieder Regierungen gegeben, denen es weniger an Entscheidungsmacht als an zündenden Ideen für (reform-)politische Gestaltung zu fehlen schien. Ideenlosigkeit gehört zweifelsohne zu den Realitäten demokratischer Regierungspolitik, die als Möglichkeit in Rechnung zu stellen ist. Empirisch besonders gut greifbar ist dieser Faktor in Kontexten mit geringen konstitutionellen bzw. institutionellen Reformhemmnissen, wie Großbritannien, in denen das Ausbleiben politischer Reformen nicht schlicht „dem System“ angelastet werden kann.

In Teilen des politischen Journalismus wird der Vorwurf der Ideenlosigkeit von Regierungen nicht selten mit dem Topos der „Machtversessenheit“" politischer Parteien verknüpft. Parteien strebten politische Ämter vor allem wegen dieser selbst und den damit verbundenen Privilegien an. ${ }^{47}$ Bei näherer Betrachtung kann dies jedoch schwerlich für die Mehrheit der politischen Parteien in den konsolidierten Demokratien behauptet werden. Die populäre Klage, dass Parteien ,allein an der Macht“ interessiert seien, wird von der Politikwissenschaft nicht bestätigt. Einige der belastbarsten empirischen Belege für das nachhaltige Interesse von Parteien und deren Spitzenrepräsentanten, auf der Grundlage der Kontrolle über politische Führungsämter auch politisch gestalten zu wollen, entstammen der international vergleichenden Koalitionsforschung. ${ }^{48}$ Office-seekingorientierte Ansätze allein können nur einen geringen Teil aller Koalitionsbildun-

46 In diesem Kontext ist daran zu erinnern, dass Reformen nicht notwendigerweise eine progressive Stoßrichtung besitzen müssen. Es ist offensichtlich, dass mit Reformen auch versucht werden kann, als unerwünscht angesehenen Wandlungsprozessen Einhalt zu gebieten oder deren Wirkungen zumindest zu entschärfen. In diesem Sinne haftet Reformen potentiell etwas Konservatives, auf Bewahrung hin Ausgerichtetes an. Ein entsprechender Reformbegriff spielt nicht nur in der Politik, sondern auch in der Politikwissenschaft eine zentrale Rolle. So formulieren etwa Blöcker, A. u.a., a.a. O., 13: „Reformpolitik ist der Prozess, in welchem ein System seine Struktur ändert, weil das Weiterbestehen in einer sich rasch wandelnden Umwelt eine neue Form erzwingt.“ Noch stärker auf das bewahrende Element konzentriert ist die Definition bei Busch, A.: Warum ist Reformpolitik in der Bundesrepublik so schwierig?, in: Jesse, E./Sandschneider, E. (Hg.): Neues Deutschland. Eine Bilanz der deutschen Wiedervereinigung, Baden-Baden, 2008, 107-124, hier 108: „Unter Reformpolitik soll [...] eine Politik verstanden werden, die bewusst und aktiv versucht, inhaltliche und/oder institutionelle Änderungen größerer Art vorzunehmen, die jedoch nicht systemverändernder, sondern systemstabilisierender Art sind.“

47 Freilich haben an der Entwicklung dieser Sichtweise auch namhafte Vertreter aus der Wissenschaft mitgewirkt, in Deutschland vermutlich niemand mehr als Hans Herbert von Arnim. Vgl. etwa Arnim, H.H.v.: Fetter Bauch regiert nicht gern: Die politische Klasse - selbstbezogen und abgehoben, München, 1997.

48 Müller, W. C.: Koalitionstheorien, in: Helms, L./Jun, U. (Hg.): Politische Theorie und Regierungslehre. Eine Einführung in die politikwissenschaftliche Institutionenlehre, Frankfurt/New York, 2004, 267-301. 
gen erklären. Kleinstmögliche Gewinnerkoalitionen (,,minimum-winning coalitions") stellen in der Praxis der europäischen Demokratien keineswegs das selbstverständliche Ergebnis des Regierungsbildungsprozesses dar. Die häufige Formierung von Regierungskoalitionen zwischen programmatisch-ideologisch benachbarten Parteien, die das Kriterium der kleinstmöglichen Gewinnerkoalition nicht erfüllen (und damit mindestens einem Koalitionspartner einen suboptimalen Anteil an der ämterbezogenen Verteilungsmasse bescheren), deutet auf das starke Interesse von Parteien, einen größtmöglichen Teil ihrer eigenen programmatischen Agenda umzusetzen. Ein policy-bezogenes Motiv zeigt sich auch bei dem erstrebten Zugriff von Parteien unterschiedlicher Parteifamilien auf bestimmte Ressorts. ${ }^{49}$

Vorausgesetzt, regierende Parteien haben eine reformpolitische Agenda: Welche Faktoren sind - außer den bereits skizzierten institutionellen Handlungskorridoren - entscheidend dafür, ob es tatsächlich zur Realisierung politischer Reformagenden kommt?

Große Bedeutung kommt ohne Zweifel der angemessenen politischen Kommunikation von Reformvorhaben zu. Die Kommunikationsempfindlichkeit der Gesellschaft und die Kommunikationsbedürftigkeit von als legitim empfundener Politik haben - länderübergreifend - zugenommen..$^{50}$ Die erfolgreiche Kommunikation von Reformen, insbesondere von unpopulären Reformen, ist mit besonderen Anforderungen verbunden, die über die gewachsenen Ansprüche an eine professionelle Regierungskommunikation in der Alltagspolitik noch ein gutes Stück weit hinausreichen. ${ }^{51}$

49 Budge, I.: Parties, Governments and Bureaucracies, in: ders. u.a.: The Politics of the New Europe: Atlantic to Urals, London, 1997, 265-290, hier 269. So kennzeichnet konservative Parteien eine klare Präferenz zugunsten der Besetzung von Ministerien, die den Bereichen innerer und äußerer Sicherheit gewidmet sind (Außenpolitik, Verteidigung, Inneres, Justiz). Sozialdemokratische und christdemokratische Parteien sind besonders an wohlfahrtsstaatlichen Politikfeldern wie Gesundheit und Soziales sowie Bildung interessiert und erstreben den Gewinn der entsprechenden Ressorts. Wie die Konservativen haben die Christdemokraten zudem häufig ein überdurchschnittliches Interesse am Landwirtschaftsbereich, der ansonsten freilich besonders von den Bauernparteien präferiert wird. Liberale sind demgegenüber in vielen Ländern vor allem an der Besetzung des Wirtschafts-, Finanz- und Justizministeriums interessiert, daneben auch an den Ressorts Bildung, Inneres und Handel. Grüne Parteien schließlich streben in besonderem Maße Ministerien wie Umweltschutz und Verbraucherschutz an.

50 Vgl. etwa Kamps, K./Nieland, J.-U.: Regieren und Kommunikation, in: dies. (Hg.): Regieren und Kommunikation: Meinungsbildung, Entscheidungsfindung und gouvernementales Kommunikationsmanagement, Köln, 2006, 7-20.

51 Vgl. Weidenfeld, W. (Hg.): Reformen kommunizieren, Gütersloh, 2007; Delhees, S. u.a.: Wohlfahrtsstaatliche Reformkommunikation, Baden-Baden, 2008. 
Werner Weidenfeld unterscheidet auf der Grundlage der Auswertung mehrerer Fallstudien zur Reformkommunikation insgesamt drei zentrale Bedingungsfaktoren reform- und strategiefähigen Regierens: eine angemessene institutionelle Verankerung von Regierungskommunikation im Regierungsapparat und innerhalb der regierenden Parteien; eine politische Sprache, die sich durch Glaubwürdigkeit, Problemangemessenheit und Inspirationspotential auszeichnet; und schließlich ein effizienter und strategischer Einsatz moderner Methoden politischer Kommunikation. ${ }^{52}$ In zahlreichen anderen Studien werden vor allem speziellere Aspekte der sprachlichen Dimension erfolgreicher bzw. erfolgversprechender Reformpolitik, der „Reformrhetorik“ von Regierungen, ausgiebiger beleuchtet. Ein besonderer Stellenwert wird dabei zu Recht immer wieder dem Faktor „Glaubwürdigkeit“ zugewiesen; er bildet im Rahmen eines Verständnisses von „Reformkommunikation als Persuasionsprozess" ${ }^{\text {“53 }}$ die zentrale Voraussetzung einer erfolgreichen Reformkommunikation. Die Glaubwürdigkeit von Reformkommunikatoren hängt ihrerseits maßgeblich davon ab, in welchem Maße ihnen Kompetenz und Vertrauenswürdigkeit zugeschrieben werden. Die Fähigkeit eines Reformakteurs, eine verbindende „große Erzählung“ zu entwickeln, die zudem Raum für dialogische Momente belässt, und das Erbringen einer Orientierungsleistung, durch die verfolgte Reformpolitiken nicht nur technokratisch, sondern auch normativ begründet werden, gilt heute ebenfalls weithin als notwendige Bedingung erfolgreicher Reformpolitik. ${ }^{54}$ Als entscheidend erscheint daneben vor allem ,die Begleitung des Reformprozesses durch eine umfassend angelegte und akzeptanzstiftende Kommunikationsstrategie, die alle Phasen des Entscheidungsprozesses abdeckt ". ${ }^{55}$

Selbst die beste Reformkommunikation bleibt in ihren Wirkungen kontextgebunden und situationsabhängig. $\mathrm{Zu}$ den erfolgsbegünstigenden Faktoren werden in der jüngeren Literatur mit großer Übereinstimmung ,externe Schocks“ und die Größe des Problemdrucks gerechnet. Sie sind offenbar geeignet, Problembe-

52 Weidenfeld, $W$ : Einführung: Politische Kommunikation in strategischer Perspektive, in: ders. (Hg.), a. a. O., 7-14, hier 9 .

53 Althoff, J.: Der Faktor Glaubwürdigkeit: Voraussetzung wirkungsvoller Reformkommunikation, in: Weidenfeld, W. (Hg.), a. a. O., 206-222, hier 206.

54 Vgl. Novy, L./Schmitz, G.P.: Lessons learned: politische Kommunikation im Wandel, in: Weidenfeld, W. (Hg.), a. a. O., 234-253.

55 Ebd., 239, Hervorhebung hinzugefügt. 
wusstsein zu schaffen und die Notwendigkeit reformpolitischen Handelns unmittelbar greifbar zu machen. ${ }^{56}$

Sehr viel umstrittener bleibt die Bedeutung des Reformtempos für erfolgreiche Reformen. Vor allem die neuseeländischen Erfahrungen der achtziger Jahre scheinen zu lehren, dass eine hohe Geschwindigkeit des reformpolitischen Prozesses ohne Rücksicht auf das Vorliegen eines breiten politisch-gesellschaftlichen Konsenses zugunsten reformpolitischer Aktionen durchschlagende Erfolge verspricht. ${ }^{57}$ Auf dieser Basis wurde, unter Berücksichtigung vor allem weiterer angelsächsischer Erfahrungen, generalisiert: „Die erfolgreiche Umsetzung von Reformen beruht [...] auf klarer Definition der Ziele, hohem Tempo ihrer Realisierung und entschiedener politischer Führung. Konsens wird gewonnen durch positive Ergebnisse von Entscheidungen. ${ }^{\text {c58 }}$

$\mathrm{Zu}$ einem entgegengesetzten Ergebnis gelangt eine Fallstudie über eine wichtige territoriale Verfassungsreform in der Schweiz. ${ }^{59}$ In ihr wird der langen zeitlichen Dauer des Reformprozesses ausdrücklich ein positiver Effekt zuerkannt. Wegweisender für weitere Forschungen über die Bedingungen erfolgreicher Verfassungs- bzw. Institutionenreformen dürften einige andere Einsichten derselben Studie sein. Die prozedurale Trennung der Diskussion über Distributionsaspekte von Entscheidungen über generelle Prinzipien und eine aktive Agenda-Gestaltung gehören demnach zu den maßgeblichen Faktoren ebenso wie der gezielte Rekurs auf bestimmte Ideen (im Falle der schweizerischen Föderalismusreform insbesondere das Subsidiaritätsprinzip und die Effizienz). ${ }^{60}$

\section{Die Zukunft der Reformfähigkeit demokratischer Systeme}

Die Notwendigkeit überwiegend restriktiver Reformen des Wohlfahrtsstaats wird den meisten konsolidierten Demokratien auf absehbare Zeit erhalten bleiben. Von einer strukturellen Minimierung der Reformbedürftigkeit kann insbesondere angesichts der fortbestehenden demographischen Herausforderungen, der sich

56 Vgl. Delhees, S. u. a. a.a.O., 137; mit empirisch-statistischer Untermauerung Wagschal, U.: Steuerpolitik und Steuerreformen im internationalen Vergleich, Münster, 2005, 382-413.

57 Vgl. Douglas, R.: Neuseeländische Lektionen, in: Frankfurter Allgemeine Zeitung v. 21.06. 2005.

58 So Oberreuter, H., a. a. O., 331.

59 Braun, D.: Constitutional Change in Switzerland, in: Publius, 39/2 (2009), 314-340.

60 Gerade auf die Bedeutung ideeller Orientierungen wurde auch in anderen Diskussionskontexten über die Reformfähigkeit demokratischer Gemeinwesen immer wieder nachdrücklich hingewiesen; so etwa bei Rebe, B.: Sind wir Gulliver? - Das Menetekel der Immobilität, in: Blöcker, A. u. a. (Hg.), a. a. O., 27-40. 
beinahe sämtliche der reifen Demokratien gegenübersehen, jedenfalls keine Rede sein. Die anstehende Bewältigung der fiskalischen Folgelasten der globalen Finanz- und Wirtschaftskrise zu Beginn des 21. Jahrhunderts dürfte die finanziellen Gestaltungsspielräume künftiger Regierungen vielmehr zusätzlich einengen und einen faktischen Zwang zu weiteren Reformen restriktiver und redistributiver Natur begründen.

Die reformpolitischen Potentiale, über die staatliche Entscheidungsakteure verfügen, sind heute in den wenigsten Ländern Europas bereits voll ausgeschöpft. Vor allem eine mögliche Optimierung der Regierungskommunikation könnte die Reformfähigkeit politischer Systeme positiv beeinflussen. Vorauszusetzen ist dabei freilich stets, dass mit Regierungsverantwortung ausgestattete Akteure tatsächlich daran interessiert sind, gesamtgesellschaftliche Probleme durch gezielte Reformen zu lösen, statt sich einem „Denken zweiter Ordnung“ hinzugeben, bei dem es nicht eigentlich um die Problemlösung selbst geht, sondern um die Frage, welche Lösungen mit welchem Ausmaß politischer Unterstützung der Wähler rechnen dürfen. ${ }^{61}$ Auch an diesem Punkt gibt es offensichtliche Bezüge zwischen den institutionellen Rahmenbedingungen des politischen Entscheidungsprozesses und dem Handlungspotential von Akteuren: Unter sonst gleichen Bedingungen erhöhen sich die Chancen, dass politische Entscheidungsakteure die Kraft und den Mut aufbringen, auch solche Reformziele und -strategien zu verfolgen, die möglicherweise erst mittel- oder langfristig sichtbare Erfolge zeitigen, mit der zeitlichen Dauer der Legislaturperiode. Mehrere Länder Westeuropas haben in den vergangenen Jahren den maximalen zeitlichen Umfang der Legislaturperiode erweitert und damit die Verfolgung einer vorausschauenden Reformpolitik mit mittel- und langfristigen Zielsetzungen strukturell begünstigt. In Deutschland wurde dieser Schritt in den vergangenen Jahren mehrfach diskutiert, ohne bislang eine politische Mehrheit gefunden zu haben.

Einige Autoren setzen große Hoffnungen auf die „deliberative Demokratie“. Deliberation erscheint gleichsam zwischen der öffentlichen Meinungsbildung und dem Regierungshandeln angesiedelt und verheißt Zugewinne sowohl auf der Ebene von input-Legitimation als auch der output-Legitimation. ${ }^{62}$ Ein empirisches Urteil hierzu steht noch aus. Unabhängig davon sind die Herausforderungen der näheren und weiteren Zukunft jedoch von einer Größenordnung, der

61 Vgl. Baecker, D.: Postheroisches Management. Ein Vademecum, Berlin, 1994, 81.

62 Vgl. Leggewie, C.: Deliberative Politik: Modebegriff oder neuer Regierungsstil?, in: Kamps, K./Nieland, J.-U. (Hg.), a. a. O., 21-53. 
schwerlich allein mit einer wie auch immer verbesserten Reformkommunikation entscheidungsrelevanter Akteure Genüge getan werden kann.

$\mathrm{Zu}$ den zentralen Strukturproblemen künftiger Reformpolitik gehört der Wandel des Staates bzw. der Staatlichkeit im Kontext strukturell veränderter reformpolitischer Herausforderungen. Die von Volker Hauff mit Blick auf die Umweltpolitik formulierte Problemanalyse trifft keineswegs nur auf diese zu: „Die Überwindung der wirtschaftlichen und sozialen Mißstände war im wesentlichen das Werk der Betroffenen: sie haben sich gewehrt, ihre Rechte durchgesetzt und im Tarifkampf eine gerechte Beteiligung am Volkseinkommen schrittweise verwirklicht. Bei der nachhaltigen Entwicklung von Wirtschaft und Gesellschaft ist die Frage nach den Betroffenen nicht so einfach zu beantworten. Die notwendigen Entscheidungen werden in viel größerem Umfang von einem starken Staat getroffen werden müssen, der in der Lage ist, divergierende Partikularinteressen zu bündeln und in ein überzeugendes Konzept einzuordnen, das sich am Gemeinwohl orientiert. “63

Hier liegt in der Tat ein grundsätzliches Problem: Der Staat des 21. Jahrhunderts hat sich gegenüber früheren Formationen nachhaltig verändert. Begriffe wie „Ausfransung“ oder „Zerfaserung“ von Staatlichkeit bestimmen die Diskussion ${ }^{64}$ Trotz aller Unterschiede in der Terminologie und der konzeptuellen Erfassung des Wandels besteht bei Betrachtern seit geraumer Zeit breite Übereinstimmung darüber, dass die Steuerungskapazitäten des Staates rückläufig sind. Das ,governance“-Paradigma verdankt nicht zuletzt dieser Einsicht seine Entstehung. ${ }^{65}$ Selbst die beispiellosen Interventionen vieler Staaten zur Bekämpfung der politisch-ökonomischen Folgen der internationalen Finanz- und Wirtschaftskrise rechtfertigen keine generelleren Hoffnungen auf eine „Rückkehr des Staates“ ${ }^{66}{ }^{6}$ Umso bedeutender erscheint es, die verbleibende Handlungsfähigkeit des

63 Vgl. Hauff, V.: Reformfähigkeit durch Lernbereitschaft und Dialog, in: Bentele, K./Reissert, B./Schettkat, R. (Hg.), a. a. O., 222-231, hier 231.

$64 \mathrm{Vgl}$. für eine breit angelegte und aktuelle Würdigung vor allem des deutschsprachigen Schrifttums Ladeur, K.-H.: Der Staat der „Gesellschaft der Netzwerke“, in: Der Staat, 48/2 (2009), 163-192.

65 Vgl. statt vieler Schuppert, G.F./Zürn, M. (Hg.): Governance in einer sich wandelnden Welt (PVSSonderheft 41/2008), Wiesbaden, 2008.

66 Leibfried, S.: Rückkehr des Staates?, in: Blätter für deutsche und internationale Politik, 53/5 (2008), 7985. 
Staates durch eine Modernisierung der Staatsorganisation ${ }^{67}$ in ihren strukturellen Grundvoraussetzungen zu sichern.

67 Vgl. hierzu die von Joachim Jens Hesse und dem Internationalen Institut für Staats- und Europawissenschaften (ISE) herausgegebene Schriftenreihe „Staatsreform in Deutschland und Europa“, Baden-Baden, $2006 \mathrm{ff}$. 